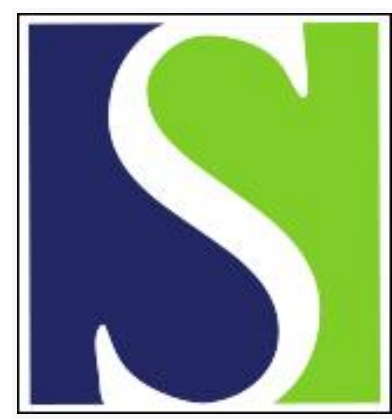

Scand J Work Environ Health 1989;15(4):271-279

https://doi.org/10.5271/sjweh.1852

Issue date: Aug 1989

Combined effects of job strain and social isolation on cardiovascular disease morbidity and mortality in a random sample of the Swedish male working population.

by Johnson JV, Hall EM, Theorell T

Affiliation: Department of Environmental Health Sciences, School of Hygiene and Public Health, Johns Hopkins University, Baltimore, Maryland 21205.

The following articles refer to this text: 2010;36(1):42-53;

2014;40(2):109-132; 2015;41(3):280-287; 2020;46(5):542-551;

2022;48(7):549-559

This article in PubMed: www.ncbi.nlm.nih.gov/pubmed/2772582 


\title{
Combined effects of job strain and social isolation on cardiovascular disease morbidity and mortality in a random sample of the Swedish male working population
}

\author{
by Jeffrey V Johnson, PhD, ${ }^{1}$ Ellen $M$ Hall, MA, ${ }^{1}$ Töres Theorell, MD, PhD ${ }^{2}$
}

\begin{abstract}
JOHNSON JV, HALL EM, THEORELL T. Combined effects of job strain and social isolation on cardiovascular disease morbidity and mortality in a random sample of the Swedish male working population. Scand J Work Environ Health 1989;15:271 - 279. This study examined the impact of psychosocial work organization on cardiovascular disease (CVD) morbidity and the nine-year cumulative mortality incidence for a random sample of the male Swedish working population $(\mathrm{N}=7219)$. A multiplicative measure was constructed to model the combined effects of psychological job demands, work-related social support and work control. Highly strained and isolated workers had a higher age-adjusted prevalence ratio for CVD morbidity and a higher age-adjusted risk ratio for CVD mortality when compared with those working under less strained and more collective conditions. Blue-collar workers showed the greatest risk for both morbidity and mortality when groups with highly isolated and strained conditions were compared to those in more collective and less strained conditions. Strained and isolated workers also had a substantially higher probability of developing and dying of CVD at a younger age than did those in less adverse environments.
\end{abstract}

Key terms: job stress, occupational health, prospective study, social support.

Cardiovascular and circulatory disorders continue to be the major causes of death in the Western world. Although much is understood about cardiovascular disease (CVD) with respect to the etiologic significance of biomedical and behavioral risk factors (such as diet, smoking, exercise, and hypertension), a significant proportion of CVD occurrence remains unexplained. In exploring the etiologic factors which might contribute to CVD, researchers have examined both environmental conditions and individual predisposing factors which may contribute to the development of cardiovascular disorders. In recent years an increasing body of literature has provided evidence that certain properties of work (such as occupational status, repetitiveness, pacing, and organizational structure) are associated with changes in cardiovascular health. For example, Buring et al (1) found, after controlling for biological risk factors, that blue-collar workers are at increased risk of CVD when compared with whitecollar workers.

In attempting to understand why an individual's occupation or the organizational characteristics of work to which he or she is exposed might contribute to the development of CVD, researchers have inves-

\footnotetext{
1 Division of Occupational Health, Department of Environmental Health Sciences, School of Hygiene and Public Health, The Johns Hopkins University, Baltimore, Maryland, United States.

2 Swedish National Institute for Psychosocial Factors and Health, Stockholm, Sweden.
}

Reprint requests to: Dr JV Johnson, Division of Occupational Health, The Johns Hopkins School of Hygiene \& Public Health, 615 N Wolfe Street, Baltimore, MD 21205, USA. tigated the content of the daily experience of worklife. It has been found, in both laboratory and field studies, that certain conditions produce measurable physiological changes. Hormonal elevations (notably the catecholamines) have been found to be associated with specific work properties (such as time pressure, loud noise, distractions and repetitiveness), and lack of control over task content and execution (2-8). For example, recent studies of prison personnel in Sweden have shown that employees in prisons with a low level of decision latitude have an elevated urinary excretion of noradrenaline (9) and also tend to have an elevated prevalence of ventricular ectopic heart beats during workhours (10).

There is additional evidence that job strain can induce excessive and long-lasting sympathoadrenal arousal, which leads to sustained blood pressure elevation $(4,8)$. Adrenaline causes the heart muscle to beat faster to increase blood flow, and other hormones cause peripheral vasoconstriction to minimize blood flow. Due to these hormonal elevations and vasoconstriction, blood pressure rises (11). In a five-year study of 380 air traffic controllers repeated measures during various job tasks demonstrated that high work load produced significant increases in blood pressure (12). These physiological reactions - if they are prolonged over an extended period of time - may result in further damage to the cardiovascular system. Theorell et al (13) found that those men who show a propensity to blood pressure elevation earlier in life and who work in "strain occupations" have much more marked blood pressure elevations at work than other men in the same 28-year-old age group. Another recently completed longitudinal study performed by Theorell and his colleagues evaluated the health of working men and 
women in six service occupations (14). Each person was examined four times. A significant positive average association between increasing job strain and increasing blood pressure at work was shown. These observations point to the possibility of a direct relationship between the psychosocial work environment and risk of heart disease (15).

We have cause to hypothesize, assuming other relevant factors to be equal, that those working for extended periods in jobs that are stressful could be expected to age more rapidly and to die earlier when they are compared to similar workers with less exposure to adversive psychosocial conditions. This connection between chronic work stress and the long-term health consequences of such exposure has been the focus of a series of investigations in the United States (1619) and Sweden (20-26). Karasek and his colleagues have predicted that biologically adversive strain will occur under a specific condition, ie, when the psychological demands of the job exceed the resources for control over task content $(20,21,23)$. A number of studies support this hypothesis and have demonstrated that workers in jobs characterized by high demands and low control are at increased risk of developing CVD (16-19, 20-26).

Johnson (27-29) and Johnson \& Hall $(30,31)$ have developed and tested an expanded version of this formulation. In investigating whether social support from co-workers is another structural factor which moderates the impact of psychological job demands, it was found that social isolation at work functions as an independent risk factor. When isolation is coupled with lack of control and high demands at work, there is a substantial increase in observed CVD prevalence risk. Thus in epidemiologic investigations there is increasing evidence that these three factors - demands, control and support - may function as a psychosocial risk complex with respect to health outcomes such as CVD.

In an effort to examine the effect of combined exposure to low control, low support, and high demands, a new variable, iso-strain (ie, combination of social isolation and job strain), was constructed in the present investigation. This composite represents the joint effects of the three job characteristics. It was constructed with the previous finding of the investigators, ie, that the three factors "demands," "control," and "support" interact in a multiplicative manner in relation to CVD prevalence risk, as the starting point (29). One of the purposes of the present study was to test this new variable on a large representative sample in an attempt to evaluate its usefulness in stress research. Because iso-strain is a univariate measure, it is a more parsimonious instrument for measuring and analyzing workplace conditions. It is easier to use and interpret in statistical analyses than the three-factor demand-control-support model with its 27 possible combinations (ie, high, medium, and low conditions for each of the factors).
This study addressed the following research questions:

1. Do workers exposed to psychosocially adverse job conditions (high iso-strain) have a greater risk for CVD morbidity and mortality than those not so exposed (low iso-strain)?

2. Do workers exposed to psychosocially adverse job conditions (high iso-strain) develop CVD at a younger age and die of CVD earlier than those exposed to more favorable work conditions (low iso-strain)?

3. Is there evidence of a dose-response relationship between the intensity of iso-strain exposure, CVD risk, and age-related trends in the development of CVD morbidity and mortality?

\section{Materials and methods}

Our study used the Swedish Central Bureau of Statistics' survey of living conditions. Data were collected in a 1-h personal interview performed by professional interviewers in 1976 and 1977. The Bureau obtained a systematic random sample from the National Register of Births in 1976 and 1977. The response rates were $80 \%$ in the two years used in our study. We used a subsample of 7219 employed men. As the measures used in this study have been extensively described elsewhere $(27-30)$, they will be discussed only briefly.

The categories of blue- and white-collar workers were defined according to the Bureau's system. Whitecollar workers were office and professional employees, and blue-collar workers were production, distribution, and service workers.

The scales for the work characteristics were based on a principal component factor analysis of the Swedish questionnaire items relating to work, which were identically worded and scored in both survey years. They appear in the appendix. The psychological job-demand indicator was constructed from two items, which asked if work was hectic and/or psychologically demanding. The work control scale measures decision-making authority, task variety, and personal freedom on the job. The social support scale consisted of five dichotomous items which measured the availability of social interaction in the workplace and whether these interactions carry over into nonworklife.

\section{Construction of the iso-strain indicator}

One of our earlier studies examined the statistical interactions of demands, control, and social support in relation to the prevalence of CVD. Using Rothman's interaction ratio technique (32-33), we found that the presence of combined exposure to all of the theoretically adverse conditions resulted in a CVD prevalence ratio that was $9 \%$ greater than that predicted in a multiplicative model. A univariate measure was constructed on the basis of the approximately multiplica- 
tive character of this interaction. We refer to this as "iso-strain" since it represents the combination of social isolation and job strain. The variable was constructed by rescoring the scales so that a high score represented an adverse condition. The three subcomponent scales were standardized to a mean of zero and a standard deviation of one. A constant term was then added to bring all scores to above one, and the three scales were multiplied together. On the basis of the frequency distribution of the resulting scale scores, the high iso-strain group was defined as the highest quintile, low iso-strain as the lowest quintile, and medium iso-strain as the middle three quintiles of the continuous indicator.

\section{Cardiovascular disease indicators}

In this study we examined two outcomes, the prevalence of CVD morbidity and cardiovascular-specific mortality over a nine-year follow-up period. The measure of CVD prevalence was based on the Swedish Statistical Bureau's health classification system, which obtained information on all types of long-term illness and disability during the personal interview with the following question concerning health status: "Do you suffer any long standing illness, effects of an injury, any disability or weakness?" Given an affirmative response, the interviewer probes: "Could you explain that a little more?" "What did the doctor say it was?', "What part of the body or organ system is affected?" The subjects were also questioned concerning their use of regular medication so that symptom-free conditions not covered by the previous questions could be included. The description of illness was coded by a central unit of the Bureau according to a coding system developed and tested by two consultant physicians. They matched reported symptoms and illness descriptions with the International Classification of Diseases, eighth revision (ICD). A general category for diseases of the cardiovascular system, developed by the Bureau, was used to classify 407 men as having CVD, a prevalence rate of $5.64 \%$. Of these cases, $93 \%$ were coded as having arteriosclerotic heart disease, cerebrovascular disease, or peripheral vascular disease (ICD codes $400-404 ; 410-414 ; 427,430-436$; and 440-445 respectively). Of the remaining $7 \%, 1.4 \%$ were coded as having a venous or pulmonary embolism or thrombosis, $2 \%$ were coded as having an ill-defined heart disease (ICD code 429), and $3.6 \%$ were coded 'as having other or unspecified forms of circulatory system disease (ICD codes 454-458). Although a small proportion of these ICD categories do not have a plausible stress-related etiology, a decision was made to use the broader CVD indicator because of the findings from prospective validation studies of the Bureau's chronic disease classification system (34). These studies had indicated that the survey-based detection system was very accurate in ascertaining broad categories of disease, such as "cardiovascular disease." As we have reported elsewhere (18) the CVD indicator used in this study was found to have good predictive and discriminant validity, for it was strongly associated with follow-up CVD mortality and not significantly associated with non-CVD mortality.

CVD mortality was assessed through the use of the official Swedish personal identification number. This number, assigned to all individuals at birth, provided the basis for linking the survey responses with mortality data from the National Death Register for the years 1976-1986. Those interviewed in 1976 were followed until 1985, and those interviewed in 1977 were followed until 1986, for a total of nine years of followup for the entire study group. We analyzed the incidence of CVD-related mortality by combining all deaths for arteriosclerotic heart disease, cerebrovascular disease, and peripheral vascular disease (ICD codes 400-404; 410-414; 427, 430-436; 440—445 respectively). In the period $1977-1986$ there were 193 deaths related to CVD. The nine-year cumulative incidence rate was $2.67 \%$.

\section{Statistical analysis}

The data were analyzed with the Statistical Analysis System (35) and a series of epidemiologic programs developed by Rothman \& Boice (36). The measures of association were the relative prevalence and relative risk ratios. The Mantel-Haenszel chi was used for hypothesis testing (37), and the confidence limits were constructed from the point estimates of the prevalence ratio $(\mathrm{PR})$ and risk ratio $(\mathrm{RR})$ and the Mantel-Haenszel test statistic with the use of test-based interval estimation (38-40). Age adjustment was performed according to Mantel-Haenszel's and Miettinen's techniques $(37-39)$.

In our examination of the change in CVD risk across the three iso-strain levels, the Mantel extension test for overall trend was calculated, stratified on age (41). The standardized risk ratio (SRR) values were computed with Miettinen's procedure, the low iso-strain group serving as the standard or reference category (42). A heterogeneity chi-square was used to examine the uniformity of effect across the age strata (36).

The logistic curve analysis for age trends was calculated according to the methods proposed by MacMahon \& Worcester (43). The adequacy of the logistic model was indicated in the goodness of fit chi-square, which in all cases was statistically nonsignificant and therefore indicated that there were no significant differences between the observed rates of CVD and those probabilities predicted by the logistic model. The logistic curves have been described by two parameters, ie, the $50 \%$ midpoint, in this analysis the year of age at which $50 \%$ of the specific iso-strain exposure group would be expected to develop CVD, and the logistic slope. By looking at the trends in CVD prevalence across various age strata, we made the assumption that age was a rough approximation for exposure time. The 
logistic curves were calculated separately for each isostrain group. Since the probability of developing and dying of CVD inevitably increases with age, our interest focused on the possibility that the manifestation of CVD would increase at a differential rate in the separate iso-strain groups.

\section{Results}

For the total study group, the workers with a high isostrain level were found to have an age-adjusted PR of 1.77 [95 \% confidence interval $(95 \% \mathrm{CI}) 1.28-2.44$ ] for CVD morbidity, when compared with the low isostrain group. Moreover, the Mantel extension test for overall trend, which included the middle category of exposure, was statistically significant $(P=0.001)$. There was a stepwise increase in risk for each increase in isostrain, ie, there was an SRR of 1.00 for the low, 1.38 for the medium, and 1.75 for the high exposure groups.

A similar and even more pronounced pattern was found among the blue-collar male sample, the high isostrain group having an age-adjusted PR of $2.04(95 \%$ CI 1.24-3.36) when compared with the low iso-strain group. The Mantel extension test for trend was statistically significant $(P=0.002)$, the $S R R$ values increasing from 1.00 for the low to 1.47 for the medium and 2.16 for the high iso-strain categories of exposure.

A different pattern was found for the white-collar sample. Those with high iso-strain had a statistically nonsignificant age-adjusted PR of 1.49 (95 \% CI $0.91-2.43$ ) when compared with those with low isostrain. The age-adjusted Mantel extension test was not statistically significant $(P=0.07)$, although the SRR values for both the medium (SRR 1.41) and high (SRR 1.34) groups were elevated above 1.00. However, since there was an indication of heterogeneity of effect across age strata, separate analyses were performed for the workers aged 44 years and younger and for those above 44 years of age. When older workers were considered separately, there was an indication of a significant trend $(P=0.03)$, and some stepwise increase in CVD prevalence risk, the medium iso-strain group having an SRR of 1.64 and the high iso-strain group an SRR of 1.74. It should be noted that the differential between the medium and high iso-strain levels for white-collar workers was not as substantial as that found for the total or blue-collar samples. The trend in the sample of younger white-collar workers, although not significant $(P=0.20)$, was actually reversed, the medium (SRR 0.88) and high iso-strain groups (SRR 0.38) having SRR values below that of the low iso-strain group.

In the analysis of the CVD mortality of the total sample, the high iso-strain group was found to have an age-adjusted RR of $1.92(95 \%$ CI 1.15-3.21) when compared with the low iso-strain category. The Mantel extension test was statistically significant $(P=0.02)$ and therefore indicated some degree of trend. However, the SRR values for the medium (SRR 1.71) and high (SRR 1.90) iso-strain categories were not markedly different.

Of the blue-collar subsample, the high iso-strain group was found to have an age-adjusted RR of 2.58 (95 \% CI 1.06-6.28) when compared with the low isostrain group. The Mantel extension test was only of borderline statistical significance $(P=0.08)$. Although the SRR for the medium iso-strain group (SRR 2.21) was elevated relative to that of the low iso-strain group, it was of a similar magnitude as the SRR of the high iso-strain group (SRR 2.54).

For the white-collar sample, the high iso-strain group had an age-adjusted RR of 1.31 (95\% CI 0.58-2.96) for CVD mortality when compared with the low isostrain group. The Mantel extension test was not statistically significant $(P=0.23)$, and the trend of effect, an SRR of 1.39 for the medium and of 1.26 for the high iso-strain group, provided no indication of a doseresponse relationship.

The results of the logistic analysis of age trends for the prevalence of CVD morbidity in the total population is displayed in figure 1 . The low iso-strain group had a markedly slower age progression of cardiovascular disease morbidity than did the other iso-strain groups. Although there was a greater magnitude of CVD morbidity at each age within the high iso-strain category, the age trend was not markedly different from that seen in the medium iso-strain group.

As in the PR, RR and SRR analyses, the analysis of age trends for CVD morbidity showed a more pronounced effect for the blue-collar workers than for either the total sample or the white-collar sample, as indicated by a comparison of figure 2 with figures 1 and 3 . There was a much stronger suggestion of a doseresponse relationship in this subgroup, as there was a stepwise increase in the age-related progression of CVD morbidity with increasing levels of iso-strain.

For the white-collar workers, as is shown in figure 3, the most marked finding was the difference in the age trends for CVD morbidity between the high and medium iso-strain group on the one hand and the low isostrain group on the other. However, there was no noticeable difference in trend between the high and medium iso-strain groups.

The logistic analysis of age trends in CVD mortality incidence for the total male population is displayed in figure 4. Although there was little difference in the age-related progression of CVD mortality between the high and medium iso-strain groups, the trends for these two groups were considerably more pronounced than that found for the low iso-strain category, which had a much slower age progression of CVD death.

A similar pattern was found among the blue-collar workers, as is shown in figure 5. Again, the most significant finding was the much slower age progression of CVD mortality in the low iso-strain group, when compared with the other two groups, which demonstrated rather similar trends. 
Finally, figure 6 shows the results of the age trends in CVD mortality for the white-collar workers. The overall difference in trends between the high and medium groups on the one hand and the low iso-strain group on the other was less pronounced than that found for the blue-collar group or for the total popu- lation. There was also a suggestion of a more rapid age progression among the workers with a high level of iso-strain than among those with a medium level. However, the standard errors for the parameters of the logistic function in the low iso-strain group among the blue-collar workers and the high iso-strain group

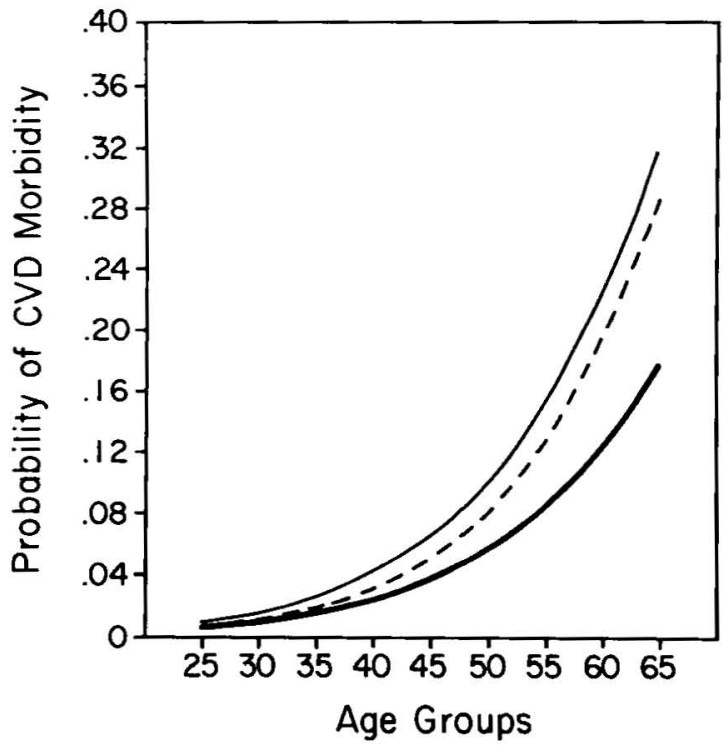

Figure 1. Logistic estimates of age trends in the prevalence of morbidity from cardiovascular disease (CVD) (number of cases 407 ) within a random sample of the total Swedish male working population $(\mathrm{N}=7219)$ by low $(-)$, medium $(---)$, and high (- ) levels of iso-strain (ie, combination of social isolation and job strain).

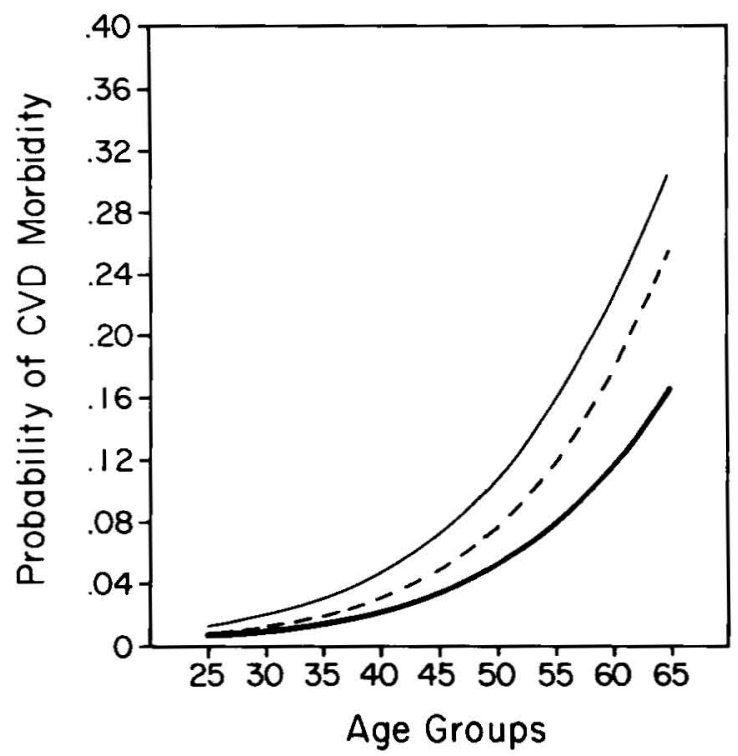

Figure 2. Logistic estimates of age trends in the prevalence of morbidity from cardiovascular disease (CVD) (number of cases 227) within a random sample of the Swedish blue-collar male working population ( $N=4235)$ by low (-), medium (-- ), and high (_- ) levels of iso-strain (ie, combination of social isolation and job strain).

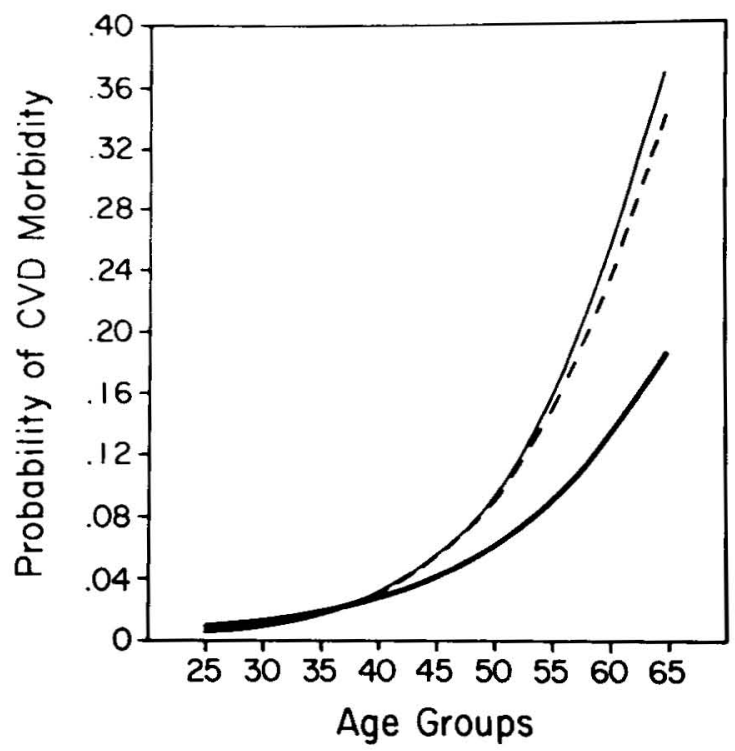

Figure 3. Logistic estimates of age trends in the prevalence of morbidity from cardiovascular disease (CVD) (number of cases $180)$ within a random sample of the Swedish white-collar male working population ( $\mathrm{N}=2984)$ by low (-), medium $(-\cdots-)$, and high $\left(-\frac{1}{-}\right)$ levels of iso-strain (ie, combination of social isolation and job strain).

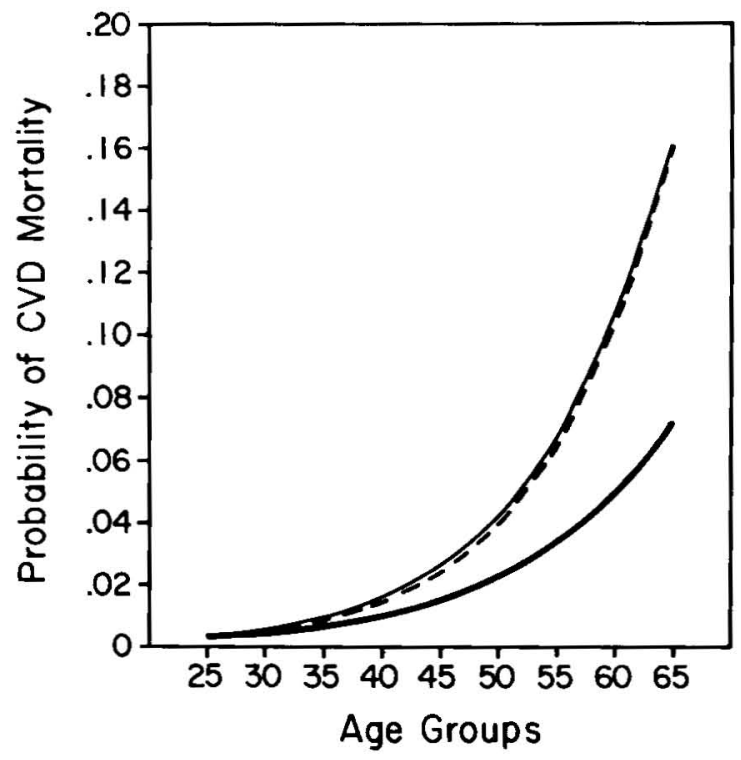

Figure 4. Logistic estimates of age trends in the nine-year incidence of mortality from cardiovascular disease (CVD) (number of deaths 193) within a random sample of the total Swedish male working population $(\mathrm{N}=7219)$ by low $(-)$, medium $(---)$, and high (_- ) levels of iso-strain (ie, combination of social isolation and job strain). 


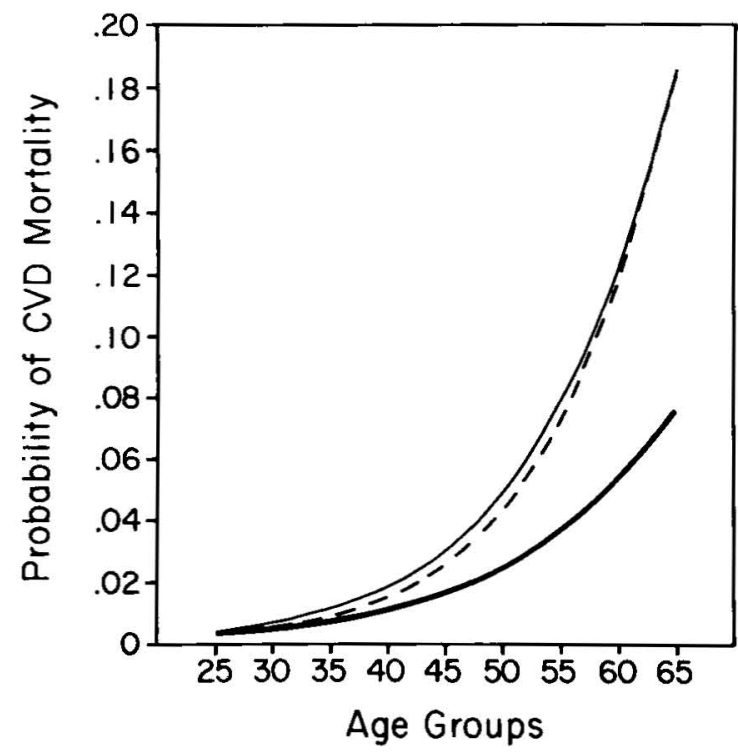

Figure 5. Logistic estimates of age trends in the nine-year incidence of mortality from cardiovascular disease (CVD) (number of deaths 122) within a random sample of the Swedish blue-collar male working population $(\mathrm{N}=4235)$ by low $(-)$, medium (- - ) , and high (_- ) levels of iso-strain (ie, combination of social isolation and job strain).

among white-collar workers were very large in the mortality analysis. Therefore these curves can only be considered approximations of possible underlying trends.

\section{Discussion}

The main finding in the present study was that the combined measure of iso-strain identified a group with remarkably slow cardiovascular aging and late CVD death risk - the low iso-strain group. These findings were consistent from morbidity to mortality and from blue-collar workers to white-collar workers. They were also consistent with the prospective findings in a previous study of Swedish men (23), among whom the absence of self-reported job strain was associated with a decreased risk of heart disease development.

The present study provides support for the hypothesis that workers exposed to adverse psychosocial work organization (represented in this study by the iso-strain variable) have an elevated risk for CVD morbidity and mortality. In terms of a dose-response relationship between iso-strain and CVD risk, the results were more uncertain. In the CVD morbidity and mortality analysis the Mantel extension test was statistically significant, and the SRR values increased with increasing levels of iso-strain in the analysis of the total sample. This same pattern was repeated in a more pronounced form for the blue-collar sample (though the Mantel extension test was only of borderline statistical significance in the mortality analysis). For the white-collar workers, however, there was only a moderate degree

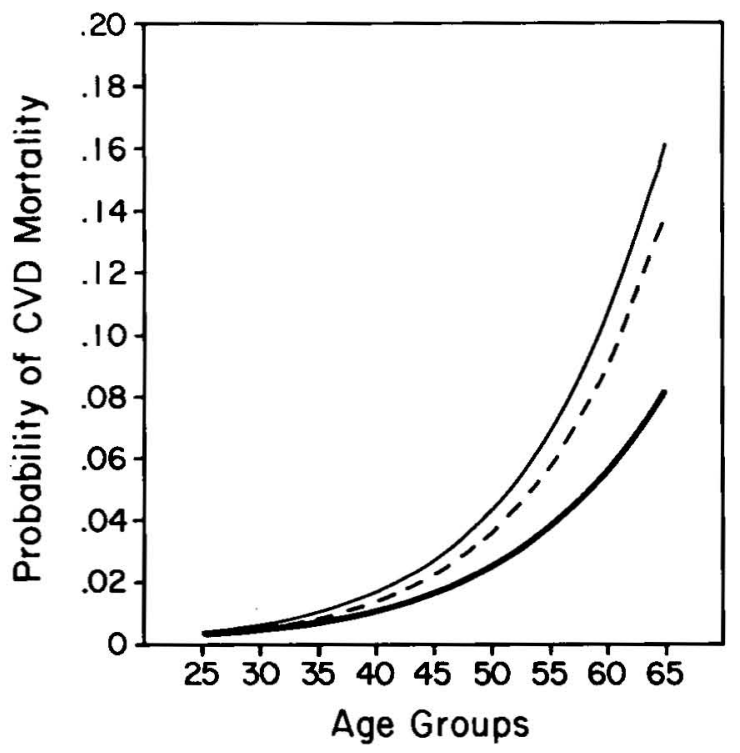

Figure 6. Logistic estimates of age trends in the nine-year incidence of mortality from cardiovascular disease (CVD) (number of deaths 71) within a random sample of the Swedish bluecollar male working population $(\mathrm{N}=2984)$ by low $(-)$, medium (-- ), and high (-_- ) levels of iso-strain (ie, combination of social isolation and job strain).

of support for a dose-response interpretation and then only for CVD morbidity in the older group.

In the logistic analysis of the age-related progression of CVD, the clearest indication of a dose-response relationship was found for CVD morbidity in the bluecollar subsample. There was a similar, if less pronounced, dose-response effect found for CVD morbidity in the total sample. For the white-collar workers, and for CVD mortality in all the groups in the study, there was relatively little, if any, indication of a stepwise increase in the age-related progression of CVD with increasing levels of iso-strain.

It is important to distinguish between the overall CVD risk magnitude represented by the age-adjusted PR, RR, and Mantel extension test, and the age-related progression of CVD. As is evident in figure 5, for example, workers with a high level of iso-strain evidenced a notably greater probability of dying of CVD from the age of 35 to 60 years than did the medium iso-strain group. This greater risk was reflected in the difference in the SRR values, 2.54 versus 2.21 . However, the trend, as represented by the logistic slopes of the two curves for these two groups, was similar. A further distinction can be made between the rate of progression of CVD (the slope of the curve) and the degree to which iso-strain accelerates the onset of the disease. As can be observed in figures 3 to 6 , although there was an identical or very similar rate of progression for the high and medium iso-strain exposure categories, progression began at an earlier age in the high iso-strain group.

Although both the overall risk of disease and the pattern of its development should be taken into ac- 
count in considerations of the effects of environmental exposure, we would argue that, in the present investigation, the pattern of age progression is perhaps more important. Though there is some indication of a doserelated acceleration of disease onset, the most marked differences were observed when the workers with either high or medium iso-strain were compared with those with low iso-strain. Rather than a clear doseresponse effect that can be attributed to levels of isostrain exposure, this study has identified a remarkably healthy group - those with low demands, high control, and high support who evidence a very slow progression of CVD morbidity and mortality.

The lack of consistent observed differences between the medium and high iso-strain categories may be due to an error in classification. The iso-strain measure may only be able to distinguish those with some exposure from those with little or no exposure. The measure may need to be refined further in order to delineate between the high and medium exposure categories.

The present study has a number of methodological strengths and weaknesses. The studied sample is fully representative of the Swedish male work force. The participation in the interviews was good. Loss to follow-up in the death register was negligible. Thus, the findings should be generalizable for Swedish working men.

CVD morbidity was studied by means of an indicator based on interviews, not on actual medical examination. Although the judgment regarding case/no case was made following the medical instructions of an expert panel, an element of self-report bias may still exist. Self-report bias can increase the risk of spurious associations since the job characteristics were also based upon self-reports. The CVD death diagnosis, on the other hand, has a relatively high degree of accuracy due to the high autopsy rate in Sweden.

When the patterns observed for the two end points are compared, there is some overlap - approximately one-third of the men who died of cardiovascular disease had been classified as "cases" in the prevalence study. The difference between the low iso-strain group and the other groups was larger for CVD mortality than for morbidity. This finding speaks in favor of a true difference. Had the CVD mortality findings been due to the workers in the case group only, they would have been weaker than the morbidity findings.

House et al (44) has pointed out another methodological concern in regard to the still common practice in the epidemiology of occupational stress to measure job characteristics at only one point in time. The subjects' reports on this single occasion may reflect a temporary phenomenon which has no significance for cardiovascular pathology. Thus it is possible that the reported associations may be spuriously weak. In a future analysis of the same samples using an occupationally based psychosocial exposure matrix, we will be able to utilize individual work histories in order to relate mortality to the duration of exposure to various categories of iso-strain.

The following explanations of the observed decreased risk of CVD among low iso-strain workers are possible:

1. The association may be spurious and simply due to the self-selection of already sick or vulnerable persons into jobs with moderate or high degrees of isostrain. This explanation seems unlikely. Sick or vulnerable persons would rather avoid than select jobs that are demanding, isolated, and uncontrollable, particularly in a country with an advanced social security system.

2. Self-report bias may have influenced the morbidity associations, but it is unlikely to explain the mortality associations. (See the preceding discussion.)

3. Accepted biomedical risk factors may have been lower in the low iso-strain group. Because of the limited information available on our sample, we were not able to examine explicitly the potential confounding effects. However, other studies $(15,30,45,46)$ have shown that biomedical risk factors do not "explain away" the association between job strain, social isolation, and CVD. Biomedical risk factors, in particular smoking habits, blood pressure, and possibly serum cholesterol, may in fact be influenced by job conditions $(45,46)$. A recent report (47) has also shown that extremely adverse job conditions may influence lipoproteins, although in general lipoproteins do not seem to be influenced to any great degree by psychosocial factors. Therefore, one important distinction should be made between variance in biomedical risk factors caused by exposure to psychosocial job conditions and biomedical risk factor variance not influenced by these job factors. Finally, psychosocial job conditions could influence illness risk directly outside the accepted biomedical risk factors.

4. The three components of iso-strain all correlate with social class. Thus, in this study, psychological job demands were significantly higher for the white-collar workers than for the blue-collar workers, while both control and support were significantly lower among the blue-collar workers. Thus the combined iso-strain measure was influenced by social class and was higher among the blue-collar workers since two of the components were predominately "blue-collar problems." There were only a few white-collar men in the high isostrain category as compared with the number of bluecollar men in this category. However, the association between reduced CVD risk and low iso-strain was not explained by the class gradient since it appeared both in the white-collar group and in the blue-collar group. Although on different levels, the white-collar workers, on the whole, had a lower risk than the blue-collar workers. These observations do, however, point to the need for a more class-specific analysis that considers 
the physical properties of work as additional potential risk factors. In a future analysis, a class-specific strategy will be introduced to our analysis of CVD risk.

\section{Acknowledgments}

This research was supported in Sweden by grant 86-119 of the Swedish Work Environment Fund and in the United States by grants NHLBI R29HL3 9633 and PHS S07RR05445.

\section{References}

1. Buring JE, Evans DA, Fiore M, Rosner B, Hennekens $\mathrm{CH}$. Occupations and risks of death from coronary heart disease. JAMA 1987;258:791-2.

2. Frankenhaeuser M. Experimental approaches to the study of human behavior as related to neuroendocrine functions. In: Levi L, ed. Society, stress and disease. London: Oxford University Press, 1971:22-36.

3. Frankenhaeuser M. Man in technological society: stress, adaptation and tolerance limits. Stockholm: University of Stockholm, 1974. (Department of Psychology research report no 26.)

4. Frankenhaeuser M. Psychoneuroendocrine approaches to the study of stressful person-environment transactions. In: Selye H, ed. Selye's guide to stress research. New York, NY: Van Nostrand Reinhold, 1980:46-70.

5. Frankenhaeuser M, Gardell B. Overload and underload in working life: outline of a multidisciplinary approach. J Human Stress 1976;2(3):35-46.

6. Frankenhaeuser M, Patkai D. Interindividual differences in catecholamine excretion during stress. Scand J Psychol 1965;6:117-23.

7. Frankenhaeuser M, Post B, Nordheden B, Sjorberg H. Physiological and subjective reactions to different physical work loads. Percept Mot Skills 1969;28:343-9.

8. Gardell B. Psychosocial aspects of industrial production methods. In: Levi L, ed. Society, stress and disease. Oxford: Oxford University Press, 1981:65-75.

9. Härenstam AB, Theorell TPG. Work conditions and urinary excretion of catecholamines - a study of prison staff in Sweden. Scand J Work Environ Health 1988; 14:257-64.

10. Härenstam A, Theorell T, Orth-Gomer K, Palm U-B, Unden A-L. Shift work, decision latitude and ventricular ectopic activity: a study of 24-hour electrocardiograms in Swedish prison personnel. Work Stress 1987;1:340-1.

11. Smith M. Occupational stress. In: Salvendy G, ed. Handbook of human factors. New York, NY: John Wiley \& Sons, 1987:844-75.

12. Rose R, Hurst M, Herd A. Cardiovascular and endocrine responses to work and the risk of psychiatric symptoms among air traffic controllers. In: Barrett J, ed. Stress and mental disorder. New York, NY: Raven Press, 1979: $101-11$.

13. Theorell T, Knox S, Svensson J, Waller D. Blood pressure variations during a working day at age 28 : effects of different types of work and blood pressure level at age 18. J Human Stress 1985;11:36-41.

14. Theorell T, Perski A, Åkerstedt T. Changes in job strain in relation to changes in physiological state: a longitudinal study. Scand J Work Environ Health 1988;14: 189-96.

15. Theorell T, Alfredsson L, Knox S, Perski A, Svensson J, Waller D. On the interplay between socioeconomic factors, personality and work environment in the pathogenesis of cardiovascular disease. Scand J Work Environ Health 1984;10:373-80.
16. Alfredsson L. Myocardial infarction and environment: use of registers in epidemiology [Dissertation]. Stockholm: Karolinska Institute, 1983.

17. Alfredsson L, Karasek R, Theorell T. Myocardial infarction risk and psychosocial work environment: an analysis of the male Swedish working force. Soc Sci Med $1982 ; 16: 463-7$.

.18. Alfredsson L, Spetz C-L, Theorell T. Type of occupation and near-future hospitalization for myocardial infarction and some other diagnosis. Int J Epidemiol 1985; $14: 378-88$.

19. Baker D. The study of stress at work. Annu Rev Public Health 1985;6:367-81.

20. Karasek R. Job content instrument: questionnaire and user's guide. Los Angeles, CA: Department of Industrial and Systems Engineering, University of Southern California, 1985.

21. Karasek R, Baker D, Marxer F, Ahlbom A, Theorell T. Job decision latitude, job demands and cardiovascular disease: a prospective study of Swedish men. Am J Public Health 1981;71:694-705.

22. Karasek R, Russell RS, Theorell T. Physiology of stress and regeneration in job related cardiovascular illness. J Human Stress 1982;8:29—42.

23. Karasek R, Theorell T, Schwartz J, Pieper C, Alfredsson L. Job, psychological factors and CHD. Adv Cardiol 1982;29:62-7.

24. Karasek R, Theorell T, Schwartz J, Schnall P, Pieper C, Michela J. Job characteristics in relation to the prevalence of myocardial infarction in the US Health Examination Survey (HES) and the Health and Nutrition Examination Survey (HANES). Am J Public Health 1988; 78(8):910-8.

25. Karasek R, Triantis KP, Chaudhry SS. Coworker and supervisor support and moderators of associations between task characteristics and mental strain. J Occup Behav 1982;3:141-60.

26. La Croix AZ. Occupational exposure to high demand/ low control work and coronary heart disease incidence in the Framingham Cohort [Dissertation]. Chapel Hill, NC: University of North Carolina, 1985.

27. Johnson JV. The impact of workplace social support and work control upon cardiovascular disease in Sweden. Stockholm: University of Stockholm, 1986. (Environmental and organizational psychology research monographs 1).

28. Johnson JV. Control, collectivity and the psychosocial work environment. In: Sauter S, Hurrell J, Cooper C, ed. Job stress and work control. London: John Wiley and Sons, 1989:54-74.

29. Johnson JV. Collective control: strategies for survival in the workplace. Int J Health Serv 1989;19:469 - 80.

30. Johnson JV, Hall EM. Job strain, work place social support and cardiovascular disease: a cross sectional study of a random sample of the Swedish working population. Am J Public Health 1988;78:1336-42.

31. Johnson JV, Hall EM. Social support in the work environment and cardiovascular disease. In: Shumaker S, Czajkowski S, ed. Social support and cardiovascular disease. New York, NY: Plenum Press (in press).

32. Rothman KJ. Synergy and antagonism in cause-effect relationships. Am J Epidemiol 1974;99:385-8.

33. Rothman K. The estimation of synergy or antagosnim. Am J Epidemiol 1976;103:506-11.

34. Magi M, Allander E, Bjelle A, Ragnarsson A. Rheumatic disorders in a health survey: how valid are the reports? Scand J Soc Med 1984;12:141-6.

35. SAS Institute Inc. SAS user's guide: basics, version 5 edition. Cary, NC: SAS Institute Inc, 1985.

36. Rothman K, Boice J Jr. Epidemiologic analysis with a programmable calculator. Boston, MA: Epidemiology Resources, 1982.

37. Mantel N, Haenszel W. Statistical aspects of the analysis of data from retrospective studies of disease. J Natl Can- 
cer Inst 1959;22:719-48.

38. Miettinen $\mathrm{O}$. Confounding and effect modification. Am 3 Epidemiol 1974;100:350-5.

39. Miettinen O. Simple interval estimation of risk ratio. Am J Epidemiol 1974;100:515-6.

40. Miettinen $O$. Estimability and estimation in case-referent studies. Am J Epidemiol 1976;103:226-35.

41. Mantel N. Chi-square tests with one degree of freedom: extensions of the Mantel-Haenzel procedure. J Am Stat Assoc 1963;58:690-700.

42. Miettinen OS. Standardization of risk ratios. Am J Epidemiol 1972;96:383-8.

43. MacMahon B, Worcester J. Age at menopause: US 1960 , 1962. Washington, DC: US Government Printing Of-

\section{Appendix}

\section{Measurement items}

\section{Psychological job demands indicator}

The following items were scored $0=$ yes, $1=$ no:

Is your job hectic?

Is your job psychologically demanding?

Guttman coefficient of reproducibility 0.92

Coefficient of scalability 0.79

II. Work control scale

The following items were scored $0=$ never, $1=$ sometimes, 2 = often:

Influence over the planning of work

Influence over the setting of the workpace

Influence over how time is used in work

Planning of workbreaks

Planning of vacations

Flexible workhours fice, 1966. (PHS publication no 1000 , series 11 , no 19.)

44. House JS, Strecher V, Metzner HL, Robbins C. Occupational stress and health among men and women in the Tecumseh Community Health Study. J Health Soc Behav $1986 ; 27: 62-72$.

45. Pieper C, LaCroix AZ, Karasek RA. The relation of psychosocial dimensions of work with coronary heart disease risk factors: a meta-analysis of five United States data bases. Am J Epidemiol 1989;129:483-94.

46. Tagliacozzo R, Vaughn S. Stress and smoking in hospital nurses. Am I Public Health 1982;72:44 -8 .

47. Siegrist J, Siegrist K, Weber I. Sociological concepts in the etiology of chronic disease: the case of ischemic heart disease. Soc Sci Med 1986;22:247-53.
Freedom to receive a phone call during workhours Freedom to receive a private visitor at work Varied task content Varied work procedures

Possibilities for on-going education as a part of the job

Cronbach's alpha 0.70

III. Work social support scale

The following items were scored $0=$ no, $1=$ yes:

Could talk to co-workers during breaks

Could leave their job to talk with co-workers

Could interact with co-workers as part of their work

Met with co-workers outside of the workplace

Had met with a co-worker during the last six months

Cronbach's alpha 0.75 